\title{
Affective agnosia: a core affective processing deficit in the alexithymia spectrum
}

Richard D. Lane ${ }^{1,2,3^{*}}$, Mark Solms ${ }^{4,5}$, Karen L. Weihs ${ }^{1,6}$, Alex Hishaw ${ }^{7,1}$ and Ryan Smith ${ }^{6}$

\begin{abstract}
Affective agnosia, an impairment in knowing how one feels emotionally, has been described as an extreme deficit in the experience and expression of emotion that may confer heightened risk for adverse medical outcomes. Alexithymia, by contrast, has been proposed as an over-arching construct that includes a spectrum of deficits of varying severity, including affective agnosia at the more severe end. This perspective has been challenged by Taylor and colleagues, who argue that the concept of affective agnosia is unnecessary. We compare these two perspectives by highlighting areas of agreement, reasons for asserting the importance of the affective agnosia concept, errors in Taylor and colleagues' critique, and measurement issues. The need for performance-based measures of the ability to mentally represent emotional states in addition to metacognitive measures is emphasized. We then draw on a previously proposed three-process model of emotional awareness that distinguishes affective response generation, conceptualization and cognitive control processes which interact to produce a variety of emotional awareness and alexithymia phenotypes - including affective agnosia. The tools for measuring these three processes, their neural substrates, the mechanisms of brain-body interactions that confer heightened risk for adverse medical outcomes, and the differential treatment implications for different kinds of deficits are described. By conceptualizing alexithymia as a spectrum of deficits, the opportunity to match specific deficit mechanisms with personalized treatment for patients will be enhanced.
\end{abstract}

Keywords: Alexithymia, Emotional awareness, Affective agnosia, Neuroscience, Medical outcome

\section{Introduction}

The concept of alexithymia has been an important advance in our understanding of how dysregulated emotions may adversely affect physical health. The term "alexithymia" was coined by Sifneos [1] with the intention of describing a deficit in the ability to identify and describe emotions that included a limitation in the ability to put emotions into words. Although there were forerunners of this concept in Ruesch's [2] "infantile personality," Alexander's [3]

\footnotetext{
* Correspondence: lane@email.arizona.edu

'Department of Psychiatry, University of Arizona, 1501 N. Campbell Ave, Tucson, AZ 85724-5002, USA

${ }^{2}$ Department of Psychology, University of Arizona, 1503 E. University Blvd, Tucson, AZ 85721, USA

Full list of author information is available at the end of the article
}

"visceral neurosis," and Marty and d'Muzan's [4] "pensée opératoire," all of which involved limitations or impairments in the ability to symbolically represent emotions, the concept of alexithymia has come to dominate the field. Much credit for this goes not only to Sifneos and Nemiah for their clinical descriptions and early research but also to Graeme Taylor and his colleagues for creating what is now the leading measure of alexithymia, called the TwentyItem Toronto Alexithymia Scale (TAS-20) [5, 6]. In fact, in a recent paper attempting to expand upon the concept of alexithymia (which we will refer to as the "source" document), we described a variant of alexithymia called "affective agnosia" [7]. In a surprising turn, Taylor and his colleagues [8] stated (in what we will refer to as the 
"rebuttal" document) that our contribution did not advance the theory, measurement or treatment of alexithymia, and that the phenomenon in question had already been described. The purpose of the present paper is to correct certain potentially misleading statements in the rebuttal document, reaffirm our view that affective agnosia is a useful and novel concept, and explain in greater detail why we believe that to be the case. This explanation requires detailed consideration of the various points made by Taylor and colleagues. After addressing these points we will present our view of the way forward in alexithymia research.

\section{Points of agreement with Taylor and colleagues}

It is not surprising that many points of agreement (both explicit and implicit) exist in the source and rebuttal documents. Each of them reflects contributions to the alexithymia literature over several decades.

1. There is consensus that alexithymia is an important clinical construct worthy of serious attention, both in terms of theory and measurement.

2. The key point that inspired the concept of affective agnosia is agreed: individuals with alexithymia may have deficits in their ability to mentally represent emotions. If someone has such a deficit, then it naturally follows that they will not be able to describe emotional experiences in words. This is consistent with Freud's [9] original formulation of agnosia. In their rebuttal, Taylor and colleagues provided an impressively erudite review of the psychoanalytic literature on the conceptualization of alexithymia including numerous mentions of mental representation deficits. We appreciate this literature review because it supports the neuroscience-based claim we were making, and provides the relevant hypothesis-generating clinical background in this area. Although we did acknowledge that the alexithymia literature includes mention of deficits in the mental representation of emotions, many relevant references were not included. We thank Taylor and colleagues for filling this gap.

3. The concept of alexithymia arose while considering how emotion and its dysregulation could contribute to physical disease $[10,11]$. We agree that the possible linkage between alexithymia and medical disorders is important for the field and that it is important to understand the mechanisms of this association.

4. Alexithymia is difficult to treat clinically [12]; advances in our ability to treat it would be very welcome.
5. Taylor and colleagues said that pensée opératoire ("operational thinking") is an essential component of alexithymia and that the concept of affective agnosia does not explicitly include it. We agree that affective agnosia does not explicitly include the concept of pensée opératoire, and we will discuss this and its implications further below.

\section{Reasons for affirming the existence of affective agnosia}

We note that many important points about the construct of affective agnosia in the source document were not contested in the rebuttal document. These include:

1. It is important to be able to identify people with severe alexithymia. The original descriptions of alexithymia by Sifneos and Nemiah involved people who not only lacked words for feelings but appeared to have "a diminution or absence of the basic human ability to experience feelings" (emphasis added) [10]. One point of disagreement has to do with whether the current leading measure of alexithymia, the TAS-20, is capable of consistently capturing this severe kind of deficit.

2. Affective agnosia makes neurocognitive sense. It refers to a deficit in the conceptualization of affective somatic/interoceptive sensations (in consideration of context) and thus an impairment in recognition. As such, we use the term in a somatic/interoceptive context in a manner that is directly parallel to the traditional use of the concept of agnosia in relation to exteroceptive sensory information, such as impairments in the ability to recognize (as opposed to simply perceive) the sensory qualities of faces, written text, sounds, colors, etc.

3. There is a plausible neuroanatomical basis for affective agnosia. Rather than being defined in purely psychological terms, we ground the construct in specified neural structures and circuitry.

4. The term agnosia has an established nosological meaning. This can be useful when communicating with medical colleagues in sister disciplines, or trainees who do not have previous experience with the concept of alexithymia or the supporting literature. Colleagues who are more familiar with existing nosological conventions may well take the term "alexithymia" to mean 'inability to read words pertaining to mood,' since alexia conventionally denotes a reading disorder.

5. To understand the relation between emotion processing (and its impairments) and physical disease it is useful if not essential to have a biological model that explains the mechanisms. If 
investigators interested in psychological and social factors that influence the course and progression of physical diseases wish to communicate with physicians and other health professionals within a conceptual framework that those individuals can understand and appreciate, it is very useful to translate psychological constructs into brain-based terms that can then explain how mind/brain phenomena result in peripheral physiological changes that influence pathophysiology [13]. Without such a translation, the explanation for how such psychological factors influence physical diseases cannot be cast in mechanistic terms. In this context, an excellent summary of the neural correlates of alexithymia has recently been published by Goërlich and Aleman [14]. This review of structural and functional imaging studies addressed the cognitive and emotional features of alexithymia, including emotional attention, experience, awareness, learning and imagery. It did not, however, directly address the mechanisms by which the brain of alexithymic individuals communicates with peripheral physiological systems to heighten risk for adverse physical disease outcomes. Moreover, this review did not highlight the distinction between the mental representation of emotional states (by the default mode network in our model) and the linguistic expression of emotion in language areas, a critical distinction corresponding to the distinction between agnosia and anomia. Importantly, our neural model of affective agnosia integrates mental representation of emotion with disease-related peripheral physiological processes, both of which involve the participation of the medial prefrontal cortex [7]. The fact that the neuroanatomical basis of affective agnosia is specified makes mechanistic research possible and associated hypotheses falsifiable.

6. Performance measures and self-report measures do not measure the same thing, and correlations between them are often low. According to Dunning and colleagues [15], in the realm of complex social skills correlations between self-report and objective measures range from 0.04 to 0.17 . This is highly relevant to the current discussion, as a leading measure of affective agnosia, the Levels of Emotional Awareness Scale (LEAS) [16], is a performance measure; in contrast, the leading measure of the parent concept of alexithymia is the TAS-20, a self-report measure $[5,6]$. The correlation between these two measures is quite low $(r=-0.12$ based on a meta-analysis of 21 studies [17]), consistent with the observations of Dunning and colleagues [15]. This low correlation is likely due at least in part to differences in measurement method. However, there are also reasons to believe the TAS-20 and LEAS do not measure the same underlying phenomenon, which is also a problem not unique to alexithymia where there are multiple measures aiming to assess the same construct. On its face, the LEAS measures the extent and sophistication of emotion concept use within cognition (i.e. what types of concepts expressed in words - individuals spontaneously use to understand the bodily sensations and behaviors of self and others in social/emotional situations). In contrast, the TAS-20 asks individuals to self-report their explicit beliefs about their ability to understand emotions and bodily sensations and their preferences for certain kinds of cognitive and emotional experiences. Thus, there is a clear content difference. Further, it is also well-known that self-reported beliefs often mismatch with objective behavioral performance in other socio-emotional domains (e.g. one can believe they have a strong ability to recognize emotions and yet perform poorly on emotion recognition tasks, or the reverse). It is unsurprising, therefore, that performance on the LEAS and TAS-20 scores can diverge. Importantly, these two individual differences could have quite different impacts on physiological and behavioral responding. For example, explicit beliefs about one's emotional capacities could plausibly influence the situations an individual chooses to enter (e.g., anticipatory anxiety and avoidance of social situations due to the belief that one will not fare well socially), whereas actual emotional capacities could more strongly influence direct social interactions (e.g., being aware of the emotions of self and others in the moment and using this information to guide physiological and behavioral responding). Following on the previous point, it is important to distinguish between a true deficit and a perceived (metacognitive belief or judgment of) deficit as described in self-report inventories. This distinction maps onto the performance vs. self-report nature of the LEAS and TAS-20, respectively. It helps to explain why Taylor and colleagues also created the clinician-rated Toronto Structured Interview for Alexithymia (TSIA) [18], which consists of a clinician's evaluation of the degree to which alexithymia is present. This, in our view, is the leading measure for objectively demonstrating that alexithymia is present.

7. Deficits in the ability to acquire, represent, and subsequently use emotion concepts within deliberative cognition, which are central to the construct of affective 
agnosia, provide foci for therapeutic intervention in treating alexithymia, which is notoriously difficult to do.

\section{Errors, misstatements and misunderstandings}

Before discussing areas of disagreement, and our reasons for disagreeing, we believe it is important to set the record straight regarding certain errors, misstatements and misunderstandings in the rebuttal.

1. Taylor and colleagues state that Freud's concept of "primal repression" involved a lack of mental representation that captured the phenomenon of affective agnosia equally well, thus rendering the latter concept unnecessary. This claim overlooks an important aspect of what Freud [19] intended by his concept of "primal repression." First and foremost, he conceptualized it as an innate process, present at the origin of every normal human mind: certain impulses were never crystalized as thinkable representations because to think such things would overwhelm the nascent ego. This instinctual core of the repressed is to be distinguished from "repression proper" which refers to mental contents that had been consciously represented at one point and then were relegated to the unconscious because of their distressing content. Second, in Freud's scheme what is repressed and not consciously represented is a thought that would trigger an emotion, not a representation of emotion per se. This contrasts significantly with our notion of affective agnosia as a deficit in the cognitive representation of affective states, under a conception of emotion that is independent of dynamic defensive processes. Moreover, it is critical to emphasize that the concept of alexithymia itself refers to a deficit; it is not a defense-based concept, although defensive processes could conceivably be activated as a result of this deficit (and some defenses could also hinder learning and lead to deficit). It would seriously undermine the concept of alexithymia to assert that the extreme expression of it on a dimensional continuum reflects any kind of defensive process. Given the premier insight of Nemiah and Sifneos [10], namely that they were observing a deficit in their patients and not a defense, Freud's concept of primal repression is antithetical to their concept of alexithymia, and therefore in our view is untenable as a substitute or equivalent concept to affective agnosia. We must conclude that the concept of affective agnosia is not rendered redundant by the concept of primal repression, and therefore has potential value.

2. Taylor and colleagues state that we asserted that the term affective agnosia should replace alexithymia. A careful reading of the source document will reveal that this is not true. Perhaps confusion arose because we asserted that the extreme form of affective agnosia anchored a continuum of severity of deficits in the mental representation of emotion. We also asserted that there was a need to differentiate between an agnosic type of alexithymia (a relative inability to recognize emotions) and an anomic type of alexithymia (a relative inability to name emotions), and that the latter would anchor another continuum of severity. Both types, we proposed, would be subsumed under the superordinate, wellestablished construct of alexithymia. We elaborate further on this below.

3. Taylor and colleagues state that we did not acknowledge that heightened negative affect with alexithymia makes sense in certain contexts. In fact, we did acknowledge that such an association made sense when the impairment in affective processing is in the mild to moderate range. For example, this association makes sense in the context of psychiatric disorders where difficulties in recognizing, understanding, and describing one's own negatively valenced affective responses can contribute to such responses being prolonged and dysregulated. However, in the context of systemic medical disorders in which the influence of reported negative emotions such as depression and anxiety on medical outcome is well established [20], it is important to be able to measure impairments in emotion processing independent of self-reported negative affect so that the contribution of such impairments can be quantified. Of course, this issue can be addressed in part by controlling for negative affect in studies using the TAS-20. However, given the distinct phenomena measured by the LEAS and TAS-20, there are additional reasons to separately examine affective agnosia with the LEAS, as the construct measured by this measure does not appear related to self-reported negative affect (note: very low LEAS scores could also potentially deter self-reported negative affect, as such individuals might tend only to report somatic sensations). We elaborate on these points further below.

4. Taylor and colleagues state that we did not distinguish between emotion and feeling. This is surprising because the distinction is central to our concept of affective agnosia. Their claim may arise because of differences in how basic terms in this area are used. The distinction in question is captured by our distinction between implicit and explicit emotional responses. "Implicit" corresponds to affective (valenced) bodily responses 
(visceromotor and somatomotor responses) that can be felt as bodily sensations but not understood, whereas "explicit" corresponds to the conceptualization of those bodily sensations as emotions (allowing description in language of discrete emotions as well as the use of emotion concept knowledge to adaptively guide further visceral and behavioral responses). Depending upon the definition used, our use of the terms "bodily sensations" (comparable to "emotion" as used by Taylor and colleagues) and "emotion conceptualization" (comparable to "feelings" as used by Taylor and colleagues) can be mapped to the implicit-explicit distinction, respectively.

5. Taylor and colleagues state that we did not acknowledge that if agnosia is present then one will also observe an inability to describe emotional experience. This association follows naturally from the concept of agnosia (after all, how can one adequately describe what one doesn't recognize?) and is mentioned in our source document (7) on page 599 (also see the figure caption on the same page). However, it is worth highlighting that individuals with associative visual agnosia can describe the perceptual qualities of what they see (e.g., they can draw a picture of the shapes and colors that they see in an image but not recognize the objects in the image). In our model, this is analogous to a preserved ability to describe valenced bodily sensations, but with a deficit in the ability to recognize those sensations under an emotion concept. We also asserted that it was important to affirm the existence of an anomia version of alexithymia in which mental representation of emotional states and thus the ability to recognize these states is intact - which would allow an individual to retain the use of emotion concept knowledge to adaptively guide behavior despite the naming deficit. Taylor and colleagues argue instead that alexithymia includes both agnosia-type and anomia-type phenomena and that a designation of subtypes is not needed. We elaborate on this important distinction below.

6. In their rebuttal, Taylor and colleagues highlighted findings that were consistent with predictions based on the alexithymia construct as evidence against findings we discussed that were counter-intuitive. It appears that our intent in citing the counterintuitive findings was misunderstood. Our intended point was to convey that the heterogeneity of findings may be indicative of the problem with self-reports, which is that they cannot determine the accuracy of an individual's beliefs about their own deficits. For example, since they are minimally correlated, the same individual may have high scores on both the TAS-20 and the LEAS somewhat paradoxically indicating granular emotion concept use in behavior along with a self-perceived deficit in doing so. Moreover, the absence of high scores on the TAS-20 is not a guarantee that an impairment does not exist (e.g., a low LEAS score could still be found with moderate probability). Thus, when one study demonstrates that psychiatric patients describe a wider range of (and more differentiated) emotions during interviews as a function of higher alexithymia scores (an unexpected finding), while another study shows the opposite (expected) finding, the latter does not necessarily nullify the former; it could instead indicate the presence of subgroups in which similar self-reported ratings on the questionnaire mean different things. This position is consistent with our view that there is value in distinguishing between more and less severe manifestations of alexithymia and that additional measures are needed. It should be noted that we are not claiming that findings with the LEAS will always agree with prediction. The two measures capture different things and the explanation for unexpected findings may not be the same.

\section{Measurement issues}

Many of the conclusions regarding the differing perspectives described above arise from the findings with the LEAS, a performance measure, and the TAS-20, a selfreport measure. It is notable that both measures have strong psychometric properties. The LEAS asks respondents to describe in writing how they and another person would feel in 20 different emotion-evoking scenarios involving two people. Scoring is based on the number and differentiation of the words used to describe feelings. The internal consistency (Cronbach's alpha $=0.88$ ), inter-rater reliability (intra-class correlation of 0.96) [21], intra-rater reliability over 2 years (Pearson correlation of $r=.99$ ) and test-retest reliability at 4 weeks (spearman brown correlation $=0.8)[22]$ are very good to excellent. Many studies support the construct validity of the LEAS in healthy volunteers, psychiatric disorders and systemic medical conditions [7]. Moreover, multiple brain imaging studies [23-29] and psychophysiological evidence [30] also support the validity of the LEAS as a measure of emotional awareness.

The TAS-20 asks respondents to rate their degree of agreement on a 5-point scale with 20 statements divided into three factors (difficulty identifying feelings, difficulty describing feelings and externally oriented thinking). The TAS-20 similarly has excellent psychometric properties in terms of internal consistency, test-retest 
reliability, factorial validity and convergent and discriminant validity in student samples and clinical populations [31].

A meta-analysis of 21 studies that used the LEAS and TAS-20 in the same participants reveals a low but statistically significant correlation between them of $r=-0.12$ [17]. As noted above, this low correlation is likely attributable both to differences in methodology (self-report vs. performance measures) and content (meta-cognitive vs. direct measures of ability). Cultural factors could also be playing a role as the correlation between the two measures in a sample of 344 Japanese university students was negligible $(r=-0.03$, NS) [32]. Although this metaanalysis did not address the relation between TAS-20 factors and the LEAS, one of the largest studies using both measures that also stratified for age, sex and socioeconomic status revealed a somewhat stronger correlation with factor 3 (externally-oriented thinking) than the other factors [33]. As discussed further below, externally oriented thinking is most plausibly associated with attentional processes in our more recently expanded "three-process model" of emotional awareness - which is the final "gatekeeper" in allowing the use of emotion concepts in conscious thought. Thus, it appears sensible that this factor correlates more strongly with the LEAS, as - even if individuals possessed granular emotion concept knowledge - they would not self-report granular emotions on the LEAS if they did not attend to internal (e.g., bodily) sources of information about emotions and use this information to guide their verbal responses. Indeed, it was only TAS-20 factor 3 that correlated with the LEAS in the Japanese student sample [32].

In the remainder of this section we review statements and positions held by Taylor and colleagues in relation to measurement with which we disagree. Below we explain why we disagree and provide evidence in support of our position.

1. A key claim by Taylor and colleagues is that the TAS-20 is the best measure of alexithymia and the LEAS does not measure alexithymia. We take issue with these claims on two primary grounds. First, the TAS-20 is a meta-cognitive measure of alexithymia, in that it assesses the respondent's beliefs about the presence or absence of their own psychological deficits. Although there are significant advantages to a simple measure of this sort (such as ease of use and short completion time), which are not to be discounted, self-assessment is not equivalent to a direct performance measure of the ability to put emotions into words, which is central to the alexithymia construct.

A second reason for disagreeing is based on findings from the only study to date known to include the TSIA (which may be taken to be the leading objective measure of alexithymia), the TAS-20, and the LEAS in 87 German university students [33]. Results showed that the TSIA-LEAS correlation was $r=-0.47$. By comparison, the original report on the development of the TSIA [18] observed TAS-20-TSIA correlations of 0.36 in a community sample of 136 participants and 0.68 in a clinical sample of 97 participants. Subsequent published studies of the TSIA-TAS-20 correlation range from 0.23 to 0.52 (0.23 [34], 0.34 [35], 0.44 [36], 0.49 [37] and 0.52 [38]). Clearly, many more studies are needed, especially in clinical populations. Nevertheless, in light of the greater content similarity of the TSIA and TAS-20, the evidence indicates comparability of the two measures relative to the gold standard of the TSIA rather than superiority of one over the other. Although the LEAS was not created to be a measure of alexithymia per se, it was developed to capture the developmental line along which impairment would give rise to alexithymia $[39,40]$ and as such is foundational to the alexithymia construct. Of course, it should be acknowledged that the TSIA is directly measuring yet another phenomenon: how a clinician perceives an individual's ability to recognize and express emotions, and their observed preference to attend to and focus thought on internal sources of emotional information. While this addresses many of the disadvantages of self-report measures in tracking the TAS-20 factors, and could also be more informed by the spontaneous use of emotion words measured by the LEAS, this approach might also fail to capture other relevant internal neuro-cognitive dynamics not directly observable in externally observable behavior within the context of a clinic. All three measures may therefore carry some uniquely valuable information.

2. Taylor and colleagues assert that the LEAS is not a measure of alexithymia because it does not explicitly include pensée opératoire, or externally oriented thinking (TAS-20 Factor 3). Recent evidence indicates that Factor 3 reflects a limitation in the ability to focus one's attention on one's own emotions rather than a general bias toward focusing attention externally [41]. These findings mean that if someone is limited in their capacity to attend to and focus on internal sources of emotional information, they would more likely be exteroceptively focused and tend not to spontaneously use granular emotion terns (as measured by the LEAS). This might be relevant in collectivist vs. individualist cultures [42] and 
consistent with the observation in Japanese students that only Factor 3 (inversely) correlated reliably with the LEAS [32]. One may conclude from this that the LEAS measures the output produced by participants who (to variable degrees) attend to their feelings, conceptualize/identify them, and then describe them, thus constituting a performance measure of the end product of the interactions between factors 3,1 , and 2 of the TAS-20, respectively, that ultimately determine an individual's tendency to exhibit conscious awareness of their emotions. As such, the LEAS is arguably measuring performance that depends on the three skills that the TAS-20 aims to measure (beliefs about) through self-assessment.

3. Taylor and colleagues assert that the TAS-20 is a comprehensive measure of the alexithymia continuum, it covers the extremes quite adequately, and thus an independent objective measure of a severe deficit is not needed. Moreover, they assert that Factor 1 (difficulty identifying feelings) is an adequate measure of the affective agnosia phenomenon. In their rebuttal, they offered no evidence that TAS-20 Total or Factor 1 can capture severe alexithymia defined by independent objective criteria. Notably, we recently reported on a case of severe alexithymia [43] in which the TAS-20 score was very high (and the LEAS score was very low); yet, the patient involved was highly aware of her deficit, which seems to be a requirement for accurate self-ratings on the TAS-20. Since the TAS-20 is a meta-cognitive measure and the LEAS is a performance measure, it would seem potentially useful to have both types of measures available given the low correlation between the two (i.e., especially in cases of low awareness of deficit in the presence of severe deficit, which could result in both low TAS-20 scores and low LEAS scores).

Taylor and colleagues argued that our study using "theory of mind" measures in relation to the TAS-20 and LEAS in patients with somatoform disorders, conversion disorders, and medical disorders with somatic symptoms [44] was not relevant to the current discussion because cognitive theory of mind measures are independent of theory of mind related to emotional states. First, this is a debatable point. For example, large-scale neural networks associated with conceptualization and internal simulation processes, such as the default network, appear to do so in a domain-general manner [45] - which would suggest at least partially overlapping mechanisms contributing to mental representation of emotional states, self-related cognition, and cognitive theory of mind.
Further, current distinctions in the literature between "affective" and "cognitive" theory of mind for emotions (roughly corresponding to empathic sharing of another's emotions vs. being aware of their emotions, respectively) have conceptually distinct relations to emotional awareness - with the latter being more directly linked to the ability to report awareness of others' emotions [44].

We would also highlight that the "Reading the Mind in the Eyes Test" [46] included in that study involves the ability to accurately identify affective states from photos restricted to the eyes. In that study, the LEAS correlated positively and significantly with the Eyes Test, even after controlling for self-reported positive and negative affect on the Positive and Negative Affect Schedule (PANAS) measure [47]. By contrast, while the zero-order correlation of the TAS-20 with the Eyes Test was significant, this significant correlation disappeared when controlling for positive and negative affect. Controlling for self-reported affect is a way to isolate variance due to difficulty in identifying and describing emotional states. These findings support the conclusion that the LEAS is of value in measuring impairment in the ability to mentalize emotional states and suggests that the TAS-20 alone may not be sufficient.

Taylor and colleagues argue that because alexithymia is a continuum, there is no reason to designate a separate concept for individuals at the extreme end of severity. Here we introduce a concept that we will elaborate upon below, which is that we believe that alexithymia, conceived fundamentally as a deficit in naming and mentally representing emotion, is actually a broad clinical phenomenon that encompasses a variety of different possible mechanisms that can generate phenotypically similar clinical manifestations. If one considers the analogy of hyperglycemia as a continuum, patients can manifest the full range of fasting blood glucose levels, but this continuum is not inconsistent with the fact that categorically different mechanisms involving insufficient insulin activity, Type 1 and Type 2 diabetes, can be at fault. Similarly, we argue below for intensified efforts to understand the various neural/cognitive mechanisms that lead to the phenotypic expression of what is called alexithymia.

4. As noted above, Taylor and colleagues claim that the covariation of TAS-20 and self-reported negative affect is not a problem. They point out that alexithymia involves dysregulated affect and as such should be expected to be associated with indicators of affective distress such as anxiety and depression. 
As we noted above, this is a valid point, but it is problematic in the context of examining the association between alexithymia and medical disorders, where self-reported negative affect is already a known predictor of adverse medical outcome [20]. The concept of affective agnosia specifically captures the phenomenon that affective arousal that is persistent and undifferentiated may not be recognized as an emotional state and thus may not be reported as such (which at the extreme could reduce self-reported negative affect). Thus, it considers that arousal can still be present even though low self-reported affect would suggest that such physiological arousal is absent.

In addition to the findings regarding the Reading the Mind in the Eyes Test noted above, to date there are four other studies comparing clinical and control groups demonstrating significant group differences in TAS-20 scores in the predicted direction, but these differences in TAS-20 scores disappeared when controlling for self-reported negative affect. By contrast, comparable significant differences in these groups were observed with the LEAS that did not change when controlling for negative affect. For example, patients with essential hypertension were compared to patients with secondary hypertension due to other medical causes such as renal disease; patients with essential hypertension had higher TAS-20 and lower LEAS scores than the comparison group; when these differences were controlled for negative affect, the group difference in TAS-20 scores disappeared whereas the group difference in LEAS remained statistically significant [48]. The very same pattern was observed in three additional studies comparing patients to healthy volunteers in separate clinical contexts including skin-restricted lupus erythematosus [49], eating disorders [50] and those receiving treatment on a psychosomatic medicine inpatient treatment unit [51]. These results suggest that the LEAS is capable of detecting the influence of an impairment in emotion conceptualization in medical contexts, whereas the ability of the TAS-20 to do so is more uncertain and variable. Put in terms of underlying constructs, the tendency to use emotion concepts in thought (LEAS) may capture something uniquely important in these medical contexts that is not captured by self-reported beliefs pertaining to the (conceptually related) three TAS-20 factors.

5. We also disagree about the extent to which the TAS-20 is a good predictor of medical outcome in prospective studies. In the source document we stated that "research to date has provided only very limited evidence for an association between alexithymia as measured by the TAS- 20 and medical outcomes." One review [52] revealed that "no prospective studies of TAS-20 as a predictor of medical outcome have been published." Taylor and colleagues pointed out studies of the TAS-20 as a short-term predictor of pain and physical symptoms, one of which controlled for affective distress. There are also numerous cross-sectional studies using the TAS-20 in medical contexts [53], which are of considerable value as they highlight medical contexts where affective deficits may be present. To our knowledge, however, there are still no long-term prospective epidemiological studies of TAS-20 as a predictor of systemic medical disorders after correcting for emotional distress, despite the relative ease with which the TAS-20 could be included in such studies. This is significant given that we believe that a measure of severe alexithymia (affective agnosia) could be such a predictor, based on the claims put forward above. Of course, this will need to be confirmed by such prospective studies using the LEAS as well, which to our knowledge have not yet been attempted.

In their rebuttal, Taylor and colleagues highlighted results of a 20-year follow-up study that began in the late 1980s [54], in 2321 men in Finland using the version of the TAS developed prior to TAS-20, the TAS-26. Thus, the results do not address the statements made above about the TAS-20. The study in question [55] revealed that the risk of cardiovascular death increased $1.2 \%$ for every 1 point increase in TAS-26 scores after controlling for a variety of variables, including depression. While this is certainly an important finding, there are some limitations worth considering. For example, the prevalence of alexithymia in this sample was $34 \%$ compared to a $17 \%$ prevalence rate of alexithymia in Finnish men using the TAS-20 [56] - leading to potential issues regarding generalization. It is also unclear whether the higher prevalence of alexithymia could be related to 1 ) the use of the older TAS-26 measure, 2) a possible cohort effect of elevated alexithymia involving trauma related to World War II in Finnish men, or 3) the ability of older age to itself account for the high rates of alexithymia (which could be a different generational cohort effect).

Further light has been shed on the issue in a larger study in France that began in the 1990s [57]. In contrast to the Finnish study, no participants had heart disease at baseline. In this study, 5586 participants (2312 men) were followed for an average of 8.9 years. Results showed that TAS-26 
scores did not predict cardiovascular events. Thus, the generalizability of the finding in the Finnish study is open to question. Of course, it should be acknowledged that many current studies using the LEAS also have potential limitations regarding generalizability, and a greater amount of crosscultural and longitudinal studies are necessary in this field generally.

\section{Future research on affective processing deficits in alexithymia}

Since our source article on affective agnosia [7], our own thinking on this issue has advanced. In particular, we put forward the "Three-Process Model" of affective processing [58] that extends and elaborates on the concept of affective agnosia. Specifically, we proposed that there are three interrelated (but partially dissociable) neuro-cognitive processes that can contribute to individual differences in emotional awareness - affective response generation, internal state representation (conceptualization), and cognitive control processes that modulate conscious accessibility - and that each can break down in multiple ways resulting in low emotional awareness. The affective agnosia model highlighted a deficit in conceptualization, a memory-dependent process that is the basis for categorization, that in turn leads to deficits in recognition. The three-process model highlights that a lack of affective (viscero-/skeleto-motor) response generation or overly coarse-grained affective responses could also contribute to a phenotypic lack of awareness of emotional experience. The latter was highlighted in the work of Vorst and Bermond [59] who emphasized the need to include a deficit in affective arousal as a possible factor contributing to alexithymia. The third process, cognitive control, includes externally-focused attention that could be associated with a deficit in internally focused attention to emotion [41]. Deficits in this third process can also involve state-dependent constraints in the ability to hold information about emotions in working memory (i.e., constraining explicit deliberation and verbal reporting), which could occur as a result of high levels of arousal (the first process) and an associated decline in prefrontal cortex function [60]. The third process could also include avoidance strategies such as distraction or psychological defenses, as well as other attention-related factors. As such, the three-process model embraces the content addressed by the different factors of the TAS-20 and the Bermond-Vorst Alexithymia Questionnaire but does so in a way that allows for objective rather than self-report assessments.

We recently published a detailed case report of a woman with severe alexithymia using this framework that suggested contributions of each of these three factors [43]. While the LEAS can be influenced by differences in each process, other measures may allow them to be separated. For example, affective response generation can be objectively measured using peripheral physiological responses such as skin conductance or facial EMG responses as well as self-reported valence and arousal ratings to standard affect-eliciting stimuli such as International Affective System Pictures [61] (although these self-reports also depend on subsequent representation). Conceptualization ability can be measured using scales of emotional understanding [62] and emotion granularity [63]. Cognitive control can be assessed using TAS-20 Factor 3 (externally oriented attention), measures of cognitive suppression [64] and a measure of defensiveness [65] consisting of the combination of the Marlowe-Crowne [66] and Bendig Short Form of the Taylor Manifest Anxiety Scale [67]. These are simply examples and other measures in each category are certainly possible. For example, in a recent computational modeling study, we simulated dynamics within the three-process model (discussed further below) and demonstrated ways in which measures of task reaction times and neuroimaging measures could further distinguish different abnormal mechanisms [68].

An advantage of this approach is that these three domains of objective assessment can be mapped onto different brain networks. In our source paper we focused on communication between subcortical affective response generation structures, peripheral bodily states, insula-based body state representation structures (among other relevant regions such as somatosensory cortex and anterior cingulate), medial prefrontal/default mode network structures involved in conceptualization (including conceptualization of valenced bodily states), and frontal-parietal structures associated with top-down cognitive influences. Establishing such correspondences between psychological characteristics and their neuroanatomic substrates advances the opportunities to identify brain mechanisms that influence bodily states. At the same time, detailed studies of relevant psychological processes can be undertaken corresponding to each of the three processes, such as the role of episodic and semantic memories in determining how individuals consciously or unconsciously interpret/evaluate/appraise the situations they're in, which then affect the nature and granularity of affective responses [69], the role of language and memory in categorization and conceptualization [70], and the ways that attention can be deployed or redirected to enhance or avoid emotion experiencing [71]. Based on the principle that autonomic regulation is under hierarchical control [72], the relative contribution of each level of deficit could influence the bodily expression, pathophysiological potential and disease risk for any given individual. As described in our source paper, this is consistent with a specific model whereby the mental representation deficit associated with affective agnosia involves impairment or lack of engagement of the medial prefrontal cortex; relative inactivity in this region is plausibly related to reductions in parasympathetic influence (as 
indexed by heart rate variability) which can in turn disinhibit inflammatory disease-promoting processes [73]. This framework could also potentially make it possible to identify individuals independent of self-report who phenotypically manifest affective agnosia because of a deficit in one of these processes alone or a combination of two or more.

By extension to the neurological literature, one may hypothesize the existence of additional subtypes of affective agnosia. As we discussed in the source document, a distinction is drawn in neurology between 'apperceptive' and 'associative' agnosia [74]. Apperceptive agnosia involves failure to formulate a distinct exteroceptive percept. The analog of this in our model would be a failure to have a clearly defined bodily sensation or percept mediated by the insula (and interconnected somatosensory/proprioceptive processing cortices). To our knowledge, research has not addressed variation in how well-formed bodily percepts are. One way of assessing this independent of language is to have people draw the location and contours of their bodily experiences or somatic symptoms using a cell phone-based app [75] or other tools currently used to gather data on felt distributions of bodily sensations during emotion [76]. One can also imagine provoking somatic sensations with drugs such as isoproterenol [75] or exertion and comparing the differentiation and complexity of verbal descriptions of bodily sensations in the resting/placebo vs. activated states. Associative agnosia is a disconnection disorder; it involves a normal percept "stripped of meaning." The analog of this in our model would be a failure to connect the affective bodily state to stored conceptual knowledge about it. The mechanism here would involve a failure in the functional interaction between the aforementioned structures representing bodily percepts and the medial prefrontal cortex (and associated default network structures) where conceptual knowledge about emotional states may be integrated with this perceptual information. There could also be a deficiency of stored knowledge about emotional states - which could be due to a failure to acquire emotion concept knowledge during development. Depending on its origin, this might be loosely analogous to what neurologists call 'semantic dementia.' It would be interesting, for example, to undertake a study of patients with somatic symptom disorders who appear to be overly focused on their bodily sensations. One can imagine, using this framework, that any of these possibilities might be observed in different cases. Could it be that a common problem in this context is that patients have vivid bodily sensations, and may have quite adequate stored conceptual knowledge about emotions, but fail to connect the two? The treatment strategies might be quite different depending upon what was found. All of this illustrates the heuristic potential of the affective agnosia concept.
The element of cognitive control in the three-process model also raises another very important point touched upon by Taylor and colleagues, who refer to alexithymia as a deficit in the ability to cognitively "elaborate" on experience. It is difficult to tell exactly what this means in relation to emotional experience. One interpretation is that individuals with severe alexithymia have the same emotional experience (in terms of range, differentiation and complexity) as do healthy individuals but they simply have a problem attending to or reporting on it - they in some sense "fail to formulate the words" in particular contexts despite having the conceptual resources to do so. This could represent one possible mechanism leading to the anomia version of alexithymia discussed above (however, this mechanism would be attention-dependent and could also hinder the use of emotion concept knowledge). A second interpretation is that the emotional experience and conceptualization ability of individuals with severe alexithymia is actually quite impoverished and limited relative to that of healthy individuals. This corresponds to the alternative view of Nemiah and Sifneos [10] that these patients "may lack the basic human ability to experience feelings." Thus, in the second interpretation their impairment in elaboration is a consequence of deficient internal state representation.

This second interpretation is consistent with newer constructivist views of emotion and emotional experience that hold that concepts (and the language used to refer to them) aren't mere markers of experience but are constitutive of it and able to modulate it in important ways $[70,77]$. The analogy to wine-tasting illustrates this point. Does a fine wine taste the same to a novice and an expert? Most people would say no. The expert not only has more words to describe the experience but actually recognizes more nuances in the experienced taste sensations and flavors than the novice [78]. This distinction is very important in the emotion domain as it corresponds to the distinction between agnosia and anomia. Although Taylor and colleagues, and their psychoanalytic predecessors, pointed out that alexithymic individuals lacked words and had impairments in mental representation, the key issue is that one or both could be present in any given individual - one can have anomia with or without agnosia or mental representation deficits. Therefore, it is important to make these distinctions so that accurate assessments can be made of the specific deficits that a given individual may have, and treatment strategy can be adapted accordingly. Standard treatment approaches that promote mental representation of emotion by encouraging patients to describe their feelings in a routine manner will likely be insufficient if a severe deficit of the kind described here is present.

As briefly mentioned above, we recently published a computational model $[68,79]$ and simulated how 
variations in the three processes comprising the three-process model can result in phenotypically similar deficits in emotional awareness due to different mechanisms. This basic premise is consistent with evidence that different mechanisms can lead to the same phenotype of high scores on the TAS-20 [80]. The simulation revealed that the same low emotional awareness phenotype could be due to seven distinct mechanisms. In addition, we proposed that different clinical treatment strategies could be used to address each of these distinct mechanisms. These include: 1) maladaptive affective response generation, treated by a variety of methods including cognitive therapy [81], exposure therapy [82] or acceptance and commitment therapy [83]; 2) somatically biased expectations when interpreting affective bodily sensations, treated by psychoeducation about the bodily expression of emotion or "focusing," which involves deriving the emotional meaning of bodily sensations in context [84]; 3) poor emotion concept acquisition, treated by teaching emotion concepts or emotion-focused psychotherapy [85]; 4) biased attention (failing to attend to either context or bodily sensations), treated by mindfulness-based approaches [86] or cognitive-behavioral therapy [87]; 5) high emotional volatility, treated by dialectical behavioral therapy [88] or emotion regulation therapy [89]; 6) conscious inaccessibility of emotional information, treated by overcoming cognitive avoidance tendencies [90] or psychodynamic psychotherapy (analysis of defense) [91]; and 7) reduced reflective capacity, treated by mentalization-based therapy $[92,93]$ or teaching emotion regulation strategies [89]. Note, the therapies just mentioned are just examples. It is not meant to be an exhaustive list, and many interventions could help with more than one mechanistic problem. In general, however, given that assessment methods exist to identify these mechanisms, this new perspective provides new opportunities for treating alexithymia or low emotional awareness by targeting deficits in a personalized way that can improve clinical outcomes in this area. In our view, this provides the ultimate justification for proposing the concept of affective agnosia and other variations within the alexithymia spectrum.

These considerations illustrate that there is potential utility in identifying different subtypes of alexithymia or affective agnosia that manifest in specific individuals based on objective assessment of deficits. Perhaps the concept of alexithymia could be refined and expanded to include the different types of emotion processing deficits manifested across the three processes, not captured by other well-defined syndromes, that may be present to variable degrees and perhaps in different ways in different psychiatric disorders. This is consistent with the approach taken by van der Velde and colleagues [94], in which different facets of alexithymia are thought to confer risk for different types of psychopathology. This same approach can be used to understand how different aspects of alexithymia could potentially heighten risk for various systemic medical disorders. In an intellectual environment such as this it would seem prudent to undertake large scale studies of the psychological, behavioral, and neural mechanisms in affective processing in a large number of healthy individuals and patients to develop a potentially new taxonomy of emotion processing deficits. This research could capitalize on the many advances that have been used in recent decades in the study of the mechanisms and functional consequences of alexithymia. These include, but are not limited to, structural and functional neuroimaging techniques, autonomic and other psychophysiological measures, basic cognitive functions including encoding and retrieval of episodic and semantic memories, priming and implicit processing, working memory, linguistic abilities, conceptualization and categorization skills, and executive functioning, as well as basic bodily and affective functions including interoceptive abilities, affect labeling, emotion recognition and emotion knowledge [95, 96]. Such research would also provide a context for exploring variations as a function of age, sex, socio-economic status, years of education [33], cultural/ethnic background as well as the contributions of genetics, the early childhood environment (e.g. neglect, abuse, attachment style) and their interaction to deficits of different kinds [53].

\section{Conclusion}

Taylor and colleagues are to be commended for their enormous contributions to the field of alexithymia research $[53,97]$. They have put the construct on the map in a way that would not have been possible without their conceptual and empirical work. In this paper we have endeavored to build upon the global construct of alexithymia to propose that it consists of a family of emotion processing deficits that have plausible meaning and important differential implications for alexithymia treatment and prevention. As such, by virtue of the advances that have been made, and the burgeoning of neuroscience knowledge and tools now available, we may be entering a new era of clinically-relevant neurobiologicallybased distinctions.

In this paper we have endeavored to defend and extend the concept of affective agnosia. A key concern has been the failure of the leading measure of alexithymia, the TAS-20, to predict adverse medical outcomes in long-term prospective longitudinal follow-up studies. A major reason for this may be the fact that extreme deficits in emotional experience and expression, including affective agnosia, may be difficult to capture with selfreport measures, in contrast to performance measures that assess ability or deficits directly. The distinction 
between agnosic and anomic subtypes of alexithymia led us to propose the concept of an alexithymia spectrum in which similar alexithymic or low emotional awareness phenotypes may be due to a variety of different etiologic mechanisms. We then reviewed the three-process model of emotion processing and how computational model simulations suggest that the low emotional awareness phenotype could occur due to seven distinct mechanisms, each of which has specific treatment implications. These results suggest that the traditional difficulty in treating alexithymia may derive in part from its heterogeneous nature and the need to identify the specific nature of the deficits in individual cases. The potential ability to improve patient care in this way based on this perspective is the ultimate justification for the utility of the affective agnosia concept. In addition to their application to psychiatric and neurological disorders, we would highlight the potentially critical importance of this work on the neurobiology of emotion processing deficits to the context that originally inspired the seminal contributions of Sifneos and Nemiah - namely the role of alexithymia in the development and progression of systemic medical disorders [11].

\section{Acknowledgements}

Not applicable.

\section{Authors' contributions}

RDL conceptualized the paper and wrote the first draft of the original and revised versions. MS contributed material on primal repression and the neuropsychology of agnosia. KLW and AH edited the manuscript for accuracy, clinical relevance and clinical implications. RS edited the manuscript for accuracy and added key material on the "three-process model" and its clinical and research implications. The authors read and approved the final manuscript.

\section{Funding}

Not applicable

\section{Availability of data and materials}

Not applicable.

Ethics approval and consent to participate

Not applicable.

\section{Consent for publication}

Not applicable.

\section{Competing interests}

None.

\footnotetext{
Author details

'Department of Psychiatry, University of Arizona, 1501 N. Campbell Ave, Tucson, AZ 85724-5002, USA. ${ }^{2}$ Department of Psychology, University of Arizona, 1503 E. University Blvd, Tucson, AZ 85721, USA. ${ }^{3}$ Department of Neuroscience, University of Arizona, 1040 4th St, Tucson, AZ 85721, USA. ${ }^{4}$ Department of Psychology, University of Cape Town, Rondebosch, South Africa. ${ }^{5}$ Laureate Institute for Brain Research, $6655 \mathrm{~S}$ Yale Ave, Tulsa, OK 74136, USA. ${ }^{6}$ Department of Family and Community Medicine, University of Arizona, 655 N. Alvernon Way, Suite 228, Tucson, AZ 85711, USA. ${ }^{7}$ Department of Neurology, University of Arizona, 1501 N. Campbell Ave, Tucson, AZ 85724, USA.
}

Received: 1 November 2019 Accepted: 14 April 2020

Published online: 04 September 2020

\section{References}

1. Sifneos P. Short-term psychotherapy and emotional crisis. Cambridge: Harvard University Press; 1972.

2. Ruesch J. The infantile personality. Psychosom Med. 1948;10:134-44.

3. Alexander F. Psychosomatic medicine. New York: Norton; 1950.

4. Marty P, de M'uzan M. La pensee operatoire. Rev Fr Psychoanal. 1963;27: 1345

5. Bagby R, Parker J, Taylor G. The twenty-item Toronto alexithymia scale-1. item selection and cross-validation of the factor structure. J Psychosom Res. 1994;38:23-32.

6. Bagby R, Parker J, Taylor G. The twenty-item Toronto alexithymia scale-II. Convergent, discriminant, and concurrent validity. J Psychosom Res. 1994; 38:33-40.

7. Lane RD, Weihs KL, Herring A, Hishaw A, Smith R. Affective agnosia: expansion of the alexithymia construct and a new opportunity to integrate and extend Freud's legacy. Neurosci Biobehav Rev. 2015;55:594-611.

8. Taylor GJ, Bagby RM, Parker JD. What's in the name 'alexithymia'? A commentary on "affective agnosia: expansion of the alexithymia construct and a new opportunity to integrate and extend Freud's legacy." Neurosci Biobehav Rev. 2016;68:1006-20.

9. Freud S. On aphasia; a critical study. New York: International Universities Press; 1953. (originally published 1891).

10. Nemiah J, Sifneos P. Affect and fantasy in patients with psychosomatic disorder. In: Hill O, editor. Mod Trends Psychosom Med. 1970;2:26-34.

11. Nemiah J, Freyberger $H$, Sifneos P. Alexithymia: a view of the psychosomatic process. Mod Trends Psychosom Med. 1976;3:430-9.

12. Ogrodniczuk J, Piper W, Joyce A. Effect of alexithymia on the process and outcome of psychotherapy: a programmatic review. Psychiatry Res. 2011; 190:43-8.

13. Smith R, Weihs KL, Alkozei A, Killgore WD, Lane RD. An embodied neurocomputational framework for organically integrating biopsychosocial processes: an application to the role of social support in health and disease. Psychosom Med. 2019;81(2):125-45.

14. Goërlich KS, Aleman A. In: Luminet O, Bagby RM, Taylor GJ, editors. Neuroimaging studies of alexithymia. In alexithymia: advances in research, theory, and clinical practice. Cambridge: Cambridge University Press; 2018. p. 207-49.

15. Dunning D, Heath C, Suls JM. Flawed self-assessment: implications for health, education, and the workplace. Psychol Sci Public Interest. 2004; 5(3):69-106.

16. Lane R, Quinlan D, Schwartz G, Walker P, Zeitlin S. The levels of emotional awareness scale: a cognitive-developmental measure of emotion. J Pers Assess. 1990;55:124-34

17. Maroti $D$, Lilliengren $P$, Bileviciute-Ljungar I. The relationship between alexithymia and emotional awareness: a meta-analytic review of the correlation between TAS-20 and LEAS. Front Psychol. 2018;9:453.

18. Bagby R, Taylor G, Parker J, Dickens S. The development of the Toronto structured interview for alexithymia: item selection, factor structure, reliability and concurrent validity. Psychother Psychosom. 2005;75:25-39.

19. Freud S. Inhibitions, symptoms, and anxiety. Standard. 20th ed. London: Hogarth Press; 1959. p. 77-172. (originally published 1926).

20. Lane RD. Neural substrates of implicit and explicit emotional processes: a unifyin framework for psychosomatic medicine. Psychosom Med. 2008;70(2):214-31.

21. Lane RD, Lee S, Reidel R, Weldon V, Kaszniak A, Schwartz GE. Impaired verbal and nonverbal emotion recognition in alexithymia. Psychosom Med. 1996;58(3):203-10.

22. Torrado M, Ouakinin S, Lane R. Measuring emotional awareness from a cognitive-developmental perspective: Portuguese adaptation studies of the levels of emotional awareness scale. Acta Medica Port. 2013;26(2): $145-53$.

23. Lane RD, Reiman EM, Axelrod B, Yun LS, Holmes A, Schwartz GE. Neural correlates of levels of emotional awareness: evidence of an interaction between emotion and attention in the anterior cingulate cortex. J Cogn Neurosci. 1998;10(4):525-35.

24. McRae K, Reiman EM, Fort CL, Chen K, Lane RD. Association between trait emotional awareness and dorsal anterior cingulate activity during emotion is arousal-dependent. Neuroimage. 2008;41(2):648-55. 
25. Smith R, Alkozei A, Bao J, Smith C, Lane RD, Killgore WD. Resting state functional connectivity correlates of emotional awareness. Neurolmage. 2017;159:99-106

26. Smith R, Bajaj S, Dailey NS, Alkozei A, Smith C, Sanova A, et al. Greater cortical thickness within the limbic visceromotor network predicts higher levels of trait emotional awareness. Conscious Cogn. 2018;57:54-61.

27. Smith R, Sanova A, Alkozei A, Lane RD, Killgore WD. Higher levels of trait emotional awareness are associated with more efficient global information integration throughout the brain: a graph-theoretic analysis of resting state functional connectivity. Soc Cogn Affect Neurosci. 2018; 13(7):665-75

28. Smith R, Lane RD, Sanova A, Alkozei A, Smith C, Killgore WD. Common and unique neural systems underlying the working memory maintenance of emotional vs bodily reactions to affective stimuli: the moderating role of trait emotional awareness. Front Hum Neurosci. 2018;12:370.

29. Frewen P, Lane RD, Neufeld RW, Densmore M, Stevens T, Lanius R. Neural correlates of levels of emotional awareness during trauma script-imagery in posttraumatic stress disorder. Psychosom Med. 2008;70:27-31.

30. Verkuil B, Brosschot JF, Tollenaar MS, Lane RD, Thayer JF. Prolonged nonmetabolic heart rate variability reduction as a physiological marker of psychological stress in daily life. Ann Behav Med. 2016;50(5):704-14.

31. Bagby RM, Parker JD, Taylor GJ. Twenty-five years with the 20-item Toronto alexithymia scale. J Psychosom Res. 2020;23:109940.

32. Igarashi T, Komaki G, Lane RD, Moriguchi Y, Nishimura $H$, Arakawa $H$, et al. The reliability and validity of the Japanese version of the levels of emotional awareness scale (LEAS-J). Biopsychosoc Med. 2011;5:2.

33. Lane RD, Sechrest $L$, Riedel R. Sociodemographic correlates of alexithymia. Compr Psychiatry. 1998;39(6):377-85.

34. Lichev V, Rufer M, Rosenberg N, Ihme K, Grabe HJ, Kugel H, et al. Assessing alexithymia and emotional awareness: relations between measures in a German non-clinical sample. Compr Psychiatry. 2014;55(4):952-9.

35. Inslegers R, Meganck R, Ooms E, Vanheule S, Bagby M, Taylor G, et al. The Dutch language version of the Toronto structured interview for alexithymia: reliability, concurrent validity, and factor structure. Psychol Belg. 2013;53(1):93-116.

36. Caretti V, Porcelli P, Solano L, Schimmenti A, Bagby RM, Taylor GJ. Reliability and validity of the Toronto structured interview for alexithymia in a mixed clinical and nonclinical sample from Italy. Psychiatry Res. 2011;187(3):432-6.

37. Grabe HJ, Löbel S, Dittrich D, Bagby RM, Taylor GJ, Quilty LC, et al. The German version of the Toronto structured interview for alexithymia: factor structure, reliability, and concurrent validity in a psychiatric patient sample. Compr Psychiatry. 2009;50:424-30.

38. Montebarocci O, Surcinelli P. Correlations between TSIA and TAS-20 and their relation to self-reported negative affect: a study using a multi-method approach in the assessment of alexithymia in a nonclinical sample from Italy. Psychiatry Res. 2018;270:187-93.

39. Lane R, Schwartz G. Levels of emotional awareness: a cognitivedevelopmental theory and its application to psychopathology. Am J Psychiatry. 1987;144:133-43.

40. Smith R, Quinlan D, Schwartz GE, Sanova A, Alkozei A, Lane RD. Developmental contributions to emotional awareness. J Pers Assess. 2019; 101:150-8.

41. Preece D, Becerra R, Allan A, Robinson K, Dandy J. Establishing the theoretical components of alexithymia via factor analysis: introduction and validation of the attention-appraisal model of alexithymia. Personal Individ Differ. 2017;119:341-52

42. Nisbett RE, Miyamoto Y. The influence of culture: holistic versus analytic perception. Trends Cogn Sci. 2005;9:467-73.

43. Smith R, Kaszniak AW, Katsanis J, Lane RD, Nielsen L. The importance of identifying underlying process abnormalities in alexithymia: implications of the three-process model and a single case study illustration. Conscious Cogn. 2019;68:33-46.

44. Lane RD, Hsu CH, Locke DE, Ritenbaugh C, Stonnington CM. Rol of theory of mind in emotional awareness and alexithymia: implications for conceptualization and measurement. Conscious Cogn. 2015;33:398-405.

45. Barrett LF, Satpute AB. Large-scale brain networks in affective and social neuroscience: towards an integrative functional architecture of the brain Curr Opin Neurobiol. 2013;23(3):361-72.

46. Baron-Cohen S, Wheelwright S, Hill J, Raste Y, Plumb I. The "Reading the mind in the eyes" test revised version: a study with normal adults, and adults with Asperger syndrome or high-functioning autism. J Child Psychol Psychiatry. 2001;42(2):241-51.
47. Watson D, Clark LA, Tellegen A. Positive and negative affect schedule (PANAS). J Pers Soc Psychol. 1988;54(6):1063-70.

48. Consoli SM, Lemogne C, Roch B, Laurent S, Plouin P-F, Lane RD. Differences in emotion processing in patients with essential and secondary hypertension. Am J Hypertens. 2010;23:515-21.

49. Jalenques I, Rondepierre F, Mulliez A, Lane RD, D'incan M, Consoli SM. Lower emotion awareness in skin-restricted lupus patients: a case-controlled study. Psychother Psychosom. 2018;87(5):313-5.

50. Bydlowski S, Corcos M, Jeammet P, Paterniti S, Berthoz S, Laurier C, et al. Emotion-processing deficits in eating disorders. Int J Eat Disord. 2005:37:321-9.

51. Subic-Wrana C, Bruder S, Thomas W, Lane R, Köhle K. Emotional awareness deficits in inpatients of a psychosomatic ward: a comparison of two different measures of alexithymia. Psychosom Med. 2005;67:483-9.

52. Kojima M. Alexithymia as a prognostic risk factor for health problems: a brief review of epidemiological studies. Biopsychosoc Med. 2012;6(1):21.

53. Luminet $\mathrm{O}$, Bagby RM, Taylor GJ. Alexithymia: advances in research, theory, and clinical practice. Cambridge: Cambridge University Press; 2018.

54. Kauhanen J, Kaplan G, Cohen R, Julkunen J, Salonen J. Alexithymia and risk of death in middle-aged men. J Psychosom Res. 1996;41:541-9.

55. Tolmunen T, Lehto SM, Heliste M, Kurl S, Kauhanen J. Alexithymia is associated with increased cardiovascular mortality in middle-aged Finnish men. Psychosom Med. 2010;72(2):187-91.

56. Salminen JK, Saarijärvi S, Äärelä E, Toikka T, Kauhanen J. Prevalence of alexithymia and its association with sociodemographic variables in the general population of Finland. J Psychosom Res. 1999;46(1):75-82.

57. Wiernik E, Lemogne C, Fezeu L, Arnault N, Hercberg S, Kesse-Guyot E, et al. Association between alexithymia and risk of incident cardiovascular diseases in the SUpplémentation en VItamines et Minéraux AntioXydants (SU. VI. MAX) cohort. Psychosom Med. 2018;80(5):460-7.

58. Smith R, Killgore WD, Lane RD. The structure of emotional experience and its relation to trait emotional awareness: a theoretical review. Emotion. 2018, 18(5):670

59. Vorst $\mathrm{HC}$, Bermond B. Validity and reliability of the Bermond-Vorst alexithymia questionnaire. Personal Individ Differ. 2001;30(3):413-34.

60. Thayer JF, Åhs F, Fredrikson M, Sollers JJ III, Wager TD. A meta-analysis of heart rate variability and neuroimaging studies: implications for heart rate variability as a marker of stress and health. Neurosci Biobehav Rev. 2012; 36(2):747-56.

61. Bradley MM, Lang PJ. In: Lane RD, Nadel L, editors. Measuring emotion: behavior, feeling, and physiology. In cognitive neuroscience of emotion. New York: Oxford University Press; 2000. p. 242-76.

62. Petrides KV, Mikolajczak M, Mavroveli S, Sanchez-Ruiz MJ, Furnham A, PérezGonzález JC. Developments in trait emotional intelligence research. Emot Rev. 2016:8(4):335-41.

63. Kashdan TB, Barrett LF, McKnight PE. Unpacking emotion differentiation: transforming unpleasant experience by perceiving distinctions in negativity. Curr Dir Psychol Sci. 2015;24(1):10-6.

64. Gross JJ, John OP. Individual differences in two emotion regulation processes: implications for affect, relationships, and well-being. J Pers Soc Psychol. 2003;85(2):348.

65. Lane RD, Merikangas KR, Schwartz GE, Huang SS, Prusoff BA. Inverse relationship between defensiveness and lifetime prevalence of psychiatric disorder. Am J Psychiat. 1990;147:573-8.

66. Crowne DP, Marlowe D. A new scale of social desirability independent of psychopathology. J Consult Psychol. 1960;24(4):349.

67. Bendig AW. The development of a short form of the manifest anxiety scale. J Consult Psychol. 1956;20(5):384.

68. Smith R, Lane RD, Parr T, Friston KJ. Neurocomputational mechanisms underlying emotional awareness: insights afforded by a deep temporal active inference model and their potential clinical relevance. Neurosci Biobehav Rev. 2019;107:473-91.

69. Barrett LF. How emotions are made: the secret life of the brain. Boston: Houghton Mifflin Harcourt; 2017.

70. Satpute $A B$, Nook EC, Cakar ME. The role of language in the construction of emotion and memory: a predictive coding view. In: Lane $R$, Nadel $L$, editors. Neuroscience of enduring change: applications to psychotherapy. New York: Oxford University Press; 2020. p. 56-88.

71. Lane RD, Anderson FS, Smith R. Biased competition favoring physical over emotional pain: a possible explanation for the link between early adversity and chronic pain. Psychosom Med. 2018;80:880-90. 
72. Smith R, Thayer JF, Khalsa SS, Lane RD. The hierarchical basis o neurovisceral integration. Neurosci Biobehav Rev. 2017;75:274-96.

73. Lane RD. Is it possible to bridge the biopsychosocial and biomedical models? Biopsychosoc Med. 2014;8:3-5.

74. Lissauer $\mathrm{H}$, Jackson M. A case of visual agnosia with a contribution to theory. Cogn Neuropsychol. 1988;5:157-92. (originally published in 1890).

75. Khalsa SS, Hassanpour MS, Strober MA, Craske MG, Arevian AC, Feusner JD Interoceptive anxiety and body representation in anorexia nervosa. Front Psychiatry. 2018;9:444.

76. Nummenmaa L, Glerean E, Hari R, Hietanen JK. Bodily maps of emotions. Proc Natl Acad Sci U S A. 2014;111(2):646-51.

77. Brooks JA, Shablack H, Gendron M, Satpute AB, Parrish MH, Lindquist KA. The role of language in the experience and perception of emotion: a neuroimaging meta-analysis. Soc Cogn Affect Neurosci. 2017;12(2):169-83.

78. Lane R, Pollerman B. Complexity of emotion representations. In: Feldman Barrett L, Salovey P, editors. The wisdom in feeling. New York: Guilford; 2002. p. 271-93.

79. Smith R, Parr T, Friston KJ. Simulating emotions: an active inference model of emotional state inference and emotion concept learning. Front Psychol. 2019;10:2844.

80. Ueno M, Maeda M, Komaki G. Different subgroups of high-scorers on the TAS-20 based on the big five personality traits. Personal Individ Differ. 2014; 68:71-6.

81. Beck AT. Cognitive therapy and the emotional disorders. Penguin; 1979.

82. Foa E, Hembree E, Rothbaum BO. Prolonged exposure therapy for PTSD: emotional processing of traumatic experiences therapist guide. New York: Oxford University Press; 2007.

83. Hayes SC. Get out of your mind and into your life: the new acceptance and commitment therapy. Oakland: New Harbinger Publications; 2005.

84. Gendlin ET. Focusing. New York: Bantam; 1982.

85. Greenberg LS. Emotion-focused therapy: theory and practice. Washington: APA Press; 2010.

86. Segal ZV, Teasdale JD, Williams JM. Mindfulness-based cognitive therapy: theoretical rationale and empirical status. In: Hayes S, Follette V, Linehan M, editors. Mindfulness and acceptance: expanding the cognitive-behavioral tradition. New York: Guilford; 2004. p. 45-65.

87. Craske MG. Cognitive-behavioral therapy. Washington: American Psychological Association; 2014.

88. Swales HL, Heard J, Mark G, Williams M. Linehan's dialectical behaviour therapy (DBT) for borderline personality disorder: overview and adaptation. J Ment Health. 2000;9(1):7-23.

89. Mennin DS, Fresco DM. Emotion regulation therapy. In: Gross JJ, editor. Guilford Handbook of emotion regulation, vol. 2; 2014. p. 469-90.

90. Barlow DH, Allen LB, Choate ML. Toward a unified treatment for emotional disorders-republished article. Behav Ther. 2016;47(6):838-53.

91. Brenner C. An elementary textbook of psychoanalysis. New York: Anchor; 1974.

92. Bateman A, Fonagy P. Handbook of Mentalizing in mental health practice. 2nd ed. Washington, DC: American Psychiatric Association Publishing; 2019.

93. Luyten P, Van Houdenhove B, Lemma A, Target M, Fonagy P. A mentalization-based approach to the understanding and treatment of functional somatic disorders. Psychoanal Psychother. 2012;26(2):121-40.

94. van der Velde J, Swart M, van Rijn S, van der Meer L, Wunderink L, Wiersma $D$, et al. Cognitive alexithymia is associated with the degree of risk for psychosis. PLoS One. 2015;10(6):e0124803. https://doi.org/10.1371/journal. pone.0124803

95. Luminet $\mathrm{O}$, Zamariola $\mathrm{G}$. Emotion knowledge and emotion regulation in alexithymia. In: Luminet O, Bagby RM, Taylor GJ, editors. Alexithymia: advances in research, theory, and clinical practice. Cambridge: Cambridge University Press; 2018. p. 49-77.

96. Vermeulen N, Domachowska I, Nielson KA. Memory and executive functions in alexithymia. In Luminet O, Bagby RM, Taylor GJ, editors. Alexithymia: advances in research, theory, and clinical practice. Cambridge: Cambridge University Press; 2018. p. 78-89.

97. Taylor G, Bagby R, Parker J. Disorders of affect regulation: alexithymia in medical and psychiatric illness. Cambridge: Cambridge University Press; 1997

\section{Publisher's Note}

Springer Nature remains neutral with regard to jurisdictional claims in published maps and institutional affiliations.

Ready to submit your research? Choose BMC and benefit from:

- fast, convenient online submission

- thorough peer review by experienced researchers in your field

- rapid publication on acceptance

- support for research data, including large and complex data types

- gold Open Access which fosters wider collaboration and increased citations

- maximum visibility for your research: over $100 \mathrm{M}$ website views per year

At BMC, research is always in progress.

Learn more biomedcentral.com/submissions 